\title{
An unusual case of adenoid cystic carcinoma of nasal septum mimicking as chronic sinusitis
}

\author{
Shama Shishodia ${ }^{*}$, Udit Narayan ${ }^{2}$ and Srijan Sharma ${ }^{3}$ \\ ${ }^{1}$ Wrexham Maelor Hospital, UK \\ ${ }^{2}$ Amandeep Hospital, Amritsar, Punjab, India \\ ${ }^{3}$ Pennine acute NHS trust, UK
}

\begin{abstract}
Adenoid cystic carcinoma of the nasal septum is rarely reported. Therefore, we are reporting a case in 56 years old female. Endoscopic endonasal surgical excision was done. Histology confirmed the diagnosis of adenoid cystic carcinoma of cribriform pattern. The patient has been disease-free for the last 3 years. .
\end{abstract}

\section{Introduction}

Adenoid cystic carcinomas (ACC) are malignant tumors that most commonly arise from major and minor salivary glands and accounts for approximately 3-5\% of all the head and neck carcinomas [1]. In the nasal cavity, it is exceedingly occasional but if it arises, the most common part of origin is the lateral wall of the nasal cavity. Adenoid cystic cancers are slow growing with a high propensity of local recurrence through perineural and perivascular invasion along with distant metastasis [2]. Histologically, it has been classified into three types: solid, cribriform and tubular. Surgery is the first line of treatment with radiotherapy being held in reserve for positive margin cases [1] We are describing this case of adenoid cystic carcinoma in the nasal septum, with its clinical and radiological findings in consort with its treatment.

\section{Case report}

A 56 years old woman presented to the otorhinolaryngology outpatient department with the complaint of nasal blockage and congestion for the last 18 months which was insidious in onset, initially started on the left side of the nose and then gradually progressed to both the sides. She was being treated as a case of chronic sinusitis by the physician and was only referred to otorhinolaryngology when she did not have any relief from nasal sprays, antihistamines, and frequent course of antibiotics. She also complained of difficulty in breathing, facial pain, and tenderness associated with a decreased sense of smell and recurrent episodes of epistaxis from both sides but more common on the left side. There was no history of nasal trauma, nasal discharge, postnasal drip, atopy, recurrent sinusitis, vision complaints, and nasal surgery. She had a past medical history of diabetes mellitus and hypertension. No history of asthma. She was a non-smoker and doesn't take any alcohol.

On clinical examination, bilateral nasal airway patency was decreased on both sides. Oral cavity and ear examination were normal. On anterior rhinoscopy, a firm to hard mass was visible in the posterior part of the nasal cavity on both sides. Mucosa over the mass appeared normal with no crusting, bleeding, or ulceration. On diagnostic nasal endoscopy, a bilateral reddish-pink fleshy mass was seen arising from the posterior part of nasal septum occupying the lateral wall and the floor of the nasal cavity (Figure 1). Osteomeatal units were normal on both sides. Eye examination and neck examination were nonsignificant.

A provisional diagnosis of a tumour of vascular origin was made therefore biopsy was not attempted in the outpatient department. For radiological evaluation, contrast-enhanced computed tomography scan of nose and paranasal sinus of coronal, axial and sagittal views were requested which revealed homogenous mass originating from the posterior half of the nasal septum extending into the nasal cavity bilaterally (Figures 2 and 3 ). Anteriorly nasal septum could be appreciated. Bony boundaries of bilateral maxillary, sphenoid, ethmoid and frontal sinus along with lamina papyracea seemed to be intact. Incidental finding of left concha bullosa was found. Magnetic resonance imaging showed no intracranial extension or any other soft tissue abnormality.

The surgical options of endoscopic, open approaches and radiation therapy were discussed with the patient and her relatives. She gave the informed consent for endonasal endoscopic excision of nasal mass fully aware of the fact that she might need radiotherapy in case of positive margins.

Risks and complications were explained to the patient including nasal septal perforation, recurrence, epistaxis, loss of smell sense, pain, infection, general anaesthetic risks, cerebrospinal fluid leak, vision problems, and nasal deformity. After getting the routine investigations and general anaesthetic fitness, the patient underwent endonasal endoscopic nasal mass excision with microdebrider under general

*Correspondence to: Shama Shishodia, Wrexham Maelor Hospital, UK, Tel: +91 7982487592; E-mail: drshamashishodia@gmail.com

Key words: malignant tumors, adenoid cystic carcinoma

Received: March 06, 2020; Accepted: March 20, 2020; Published: March 23 2020 
anaesthesia. Mass was removed completely leaving the perforation in the posterior part of the nasal septum (Figures 4-6). Excised mass was sent for histopathological examination which gave the final diagnosis of adenoid cystic carcinoma (ACC) of cribriform pattern with no local and distant metastasis. Excision was complete with adequate margins.

Postoperatively she had complaints of crusting and episodes of epistaxis which were relieved when she was prescribed with alkaline nasal douche and constant lubrication with Vaseline jelly. Initially, we followed up the patient monthly for 6 months with nasal endoscopy on each visit, then given her a good recovery and no signs of recurrence, we followed her up after every 2-3 months for another year. She has not

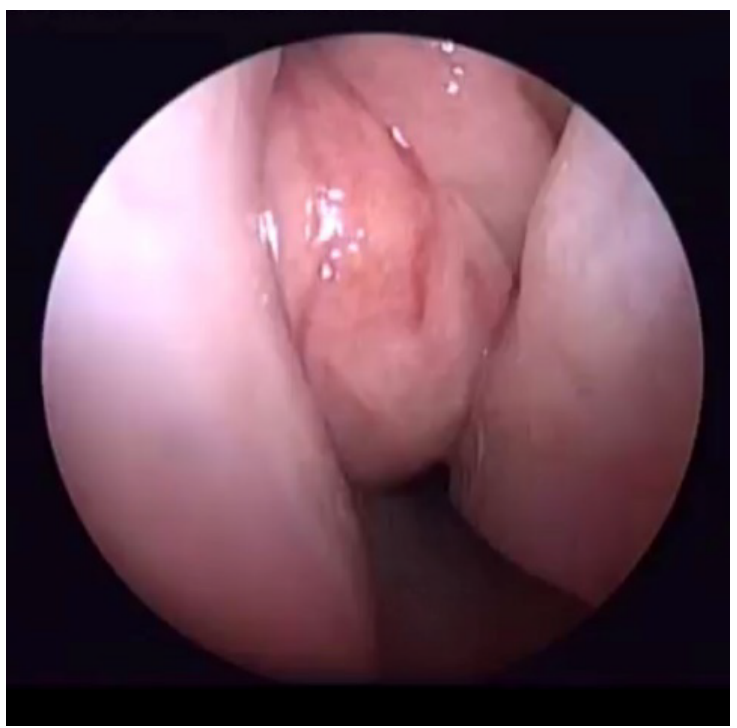

Figure 1. Endoscopic view of the nasal adenoid cystic carcinoma

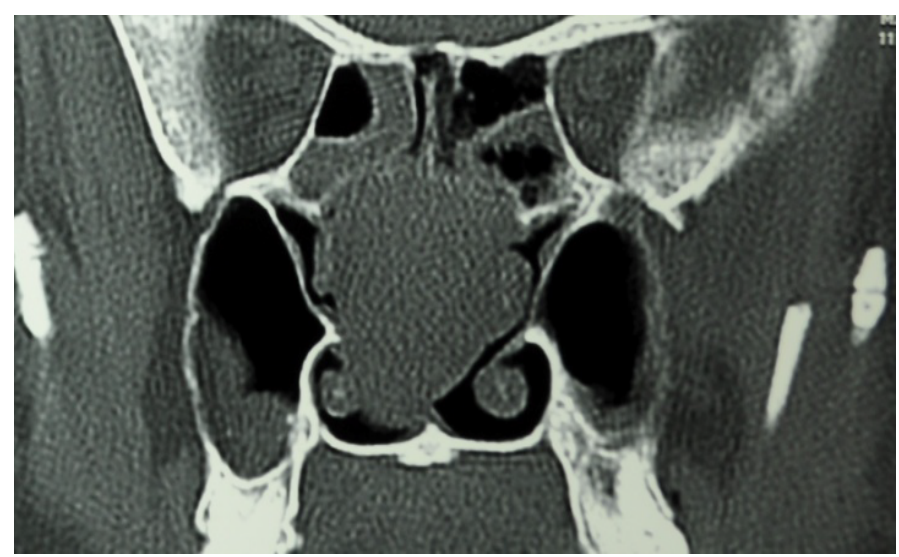

Figure 2. CT - nose and paranasal sinus (Coronal view)

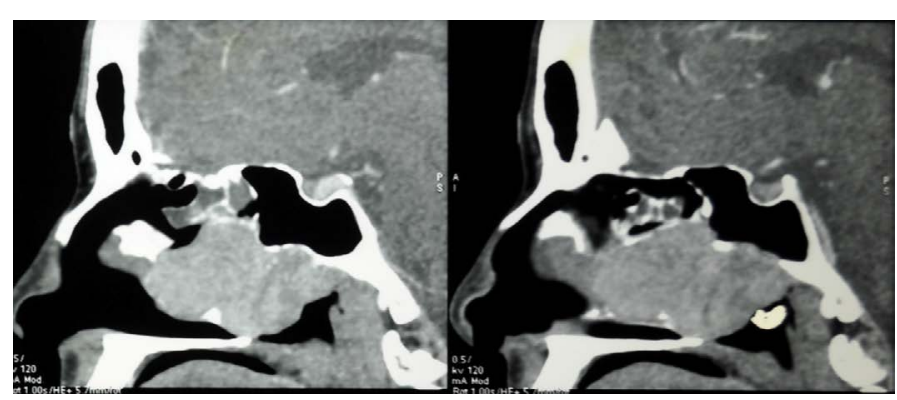

Figure 3. CT - nose and paranasal sinus (sagittal view).

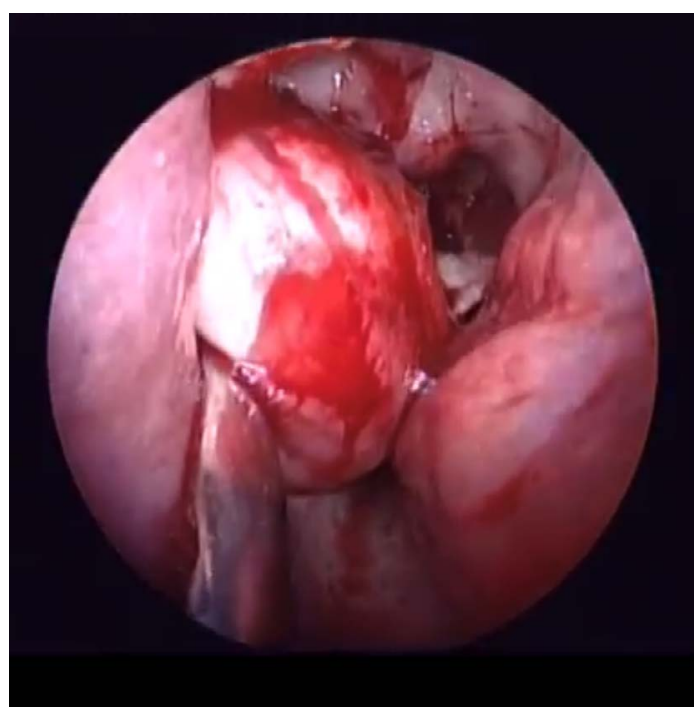

Figure 4. Endoscopic excision of nasal septal tumour

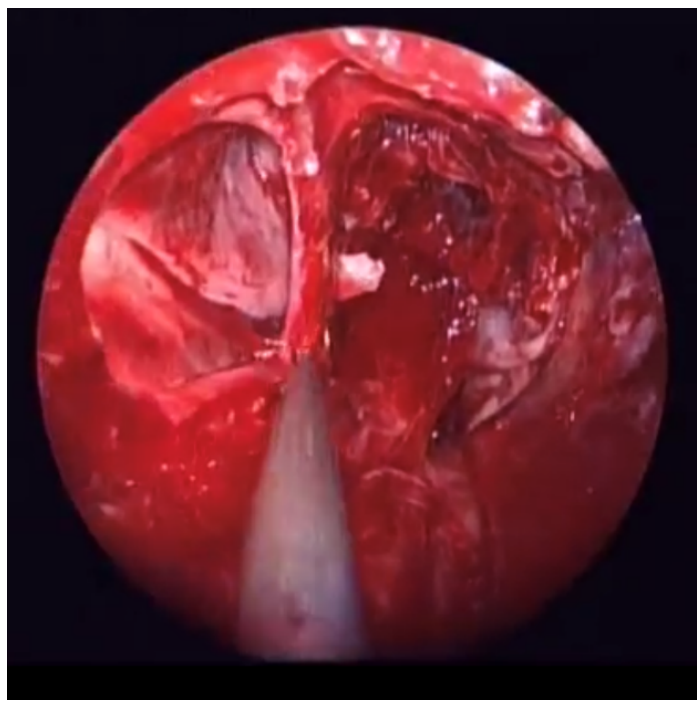

Figure 5. Posterior nasal septal perforation

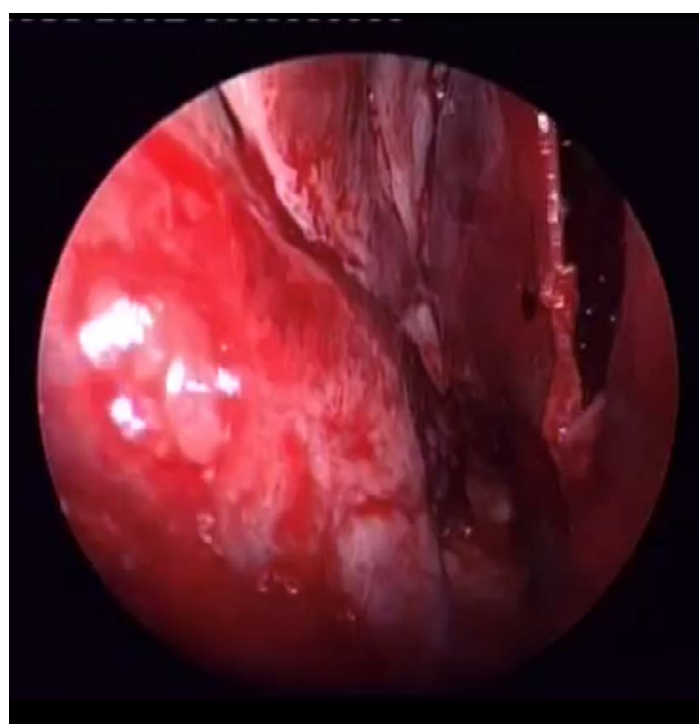

Figure 6. Nasal septal perforation 
had any symptoms and is disease-free for the last 3 years. We will be following up on the patient for 2 more years.

\section{Discussion}

Primary sinonasal tumors are rare and comprise only $3 \%$ of all head and neck cancers. They can be broadly classified into benign and malignant tumors. Benign neoplasms mainly include inverted papilloma, glomangiopericytoma, osteoma, and nerve sheath tumors while malignant tumors include squamous cell carcinoma, adenocarcinomas and adenoid cystic carcinomas [3]

ACC is of epithelial origin and has a high tendency of local recurrence and distant hematogenous metastatic spread to lungs, bone, and liver however lymphatic spread is rare. Histologically, the cribriform pattern observed in our patient is the most common type and solid type is known for its worst prognosis. Prognostic factors include tumor site, age, clinical stage, perineural invasion, histological grade, positive surgical margins, and nodal involvement. It has been reported that ACC originating from the nose and maxillary sinus are associated with poor prognosis [4]. For low-grade tumors, five-year survival rates of $85 \%$ have been seen while 10 -year survival rates irrespective of the grades of $50 \%$ have been reported [5].

ACC is more common in females of age fifty to eighty year's corresponding to the age and sex of our patient [6]. The most common symptoms include nasal obstruction, nasal congestion, facial pains, difficulty in breathing, epistaxis whereas less common symptoms include exophthalmos, visual disturbances, facial paralysis and intellectual deficit [7]. Anosmia or dysosmia is usually a late presentation but an indirect sign of tumour invasion of olfactory nerve fibers [6]. Patients usually dismiss these symptoms as they are common in other chronic conditions like chronic rhinitis, sinusitis and seek medical help at a very late stage, consequently leading to delayed management.

The surgical excision is the first modality of treatment for nasal septal malignancies. For anterior nasal septal tumors, lateral rhinotomy is the preferred surgical approach whereas, for posterior nasal tumors, sublabial incision with Denkers's approach is considered to be more appropriate [6]. ACC is considered to be radiosensitive and a total dose of 70 to 80 Gy could help in achieving local control [4]. Chemotherapy is not a standard treatment option offered to patients with limited evidence showing the role of mitoxantrone or vinorelbine alone or cisplatin and anthracycline in combination [8].

\section{Conclusion}

Even though Adenoid cystic tumors in the nasal septum is a rare entity but regardless of this fact it should be considered as one of the differential diagnosis of the nasal tumour. Also given its notorious nature of local and distant recurrence, we recommend following up a patient for a long time. Complete surgical excision should be the aim of treatment with negative margins and in case of positive and close margins, we recommend adjuvant radiotherapy.

\section{References}

1. Amit M, Binenbaum Y, Sharma K, Naomi R, Ilana R, et al. (2013) Adenoid cystic carcinoma of the nasal cavity and paranasal sinuses: A meta-analysis. $J$ Neurol Surg B Skull Base 74: 118-125. [Crossref]

2. Gill KS, Frattali MA (2015) An Unusual Presentation of Adenoid Cystic Carcinoma. $J$ Oral Maxillofac Pathol 18: 286-290. [Crossref]

3. Szewczyk-Bieda MJ, White RD, Budak MJ, Ananthakrishnan G, Brunton JN, et al (2014) A whiff of trouble: Tumours of the nasal cavity and their mimics. Clin Radiol 69: 519-528. [Crossref]

4. Ko YH, Lee MA, Hong YS, Lee SK, Jung CK, et al. (2007) Prognostic ractors affecting the clinical outcome of adenoid cystic carcinoma of the head and neck. Jpn J Clin Oncol 37: 805-811. [Crossref]

5. Priya S, Chaukar DA, Cruz AK (2011) Adenoid Cystic Carcinoma of Nasal Septum: Report of Two Cases. Int J Head Neck Surg 2:151-153.

6. Akiyama K, Karaki M, Hosikawa H, Mori N (2013) A massive adenoid cystic carcinoma of nasal septum progressed into the skull base. Auris Nasus Larynx 40: 239242.

7. Tummidi S, Kothari K, Patil R, Singhal SS, Shah V (2018) Sinonasal adenoid cystic carcinoma-role of on-site FNAC: A case report. BMC Ear Nose Throat Disord doi: 18: 6. [Crossref]

8. Ouyang DQ, Liang L, Zheng G, Ke Z, Weng G, et al. (2017) Risk factors and prognosis for salivary gland adenoid cystic carcinoma in southern china. Medicine (Baltimore) 96: e5964. [Crossref]

Copyright: (C2020 Shishodia S. This is an open-access article distributed under the terms of the Creative Commons Attribution License, which permits unrestricted use, distribution, and reproduction in any medium, provided the original author and source are credited. 